\title{
Comparison of Discrete Curvature Estimators and Application to Corner Detection
}

\author{
B. Kerautret ${ }^{1}$, J.-O Lachaud ${ }^{2}$, and B. Naegel ${ }^{1}{ }^{\star}$ \\ 1 LORIA, Nancy-University - IUT de Saint Dié des Vosges \\ 54506 Vandœuvre -lès-Nancy Cedex \\ \{kerautre, naegelbe\}@loria.fr \\ 2 LAMA, University of Savoie \\ 73376 Le Bourget du Lac \\ jacques-olivier.lachaud@univ-savoie.fr
}

\begin{abstract}
Several curvature estimators along digital contours were proposed in recent works [1-3]. These estimators are adapted to non perfect digitization process and can process noisy contours. In this paper, we compare and analyse the performances of these estimators on several types of contours and we measure execution time on both perfect and noisy shapes. In a second part, we evaluate these estimators in the context of corner detection. Finally to evaluate the performance of a non curvature based approach, we compare the results with a morphological corner detector [4].
\end{abstract}

\section{Introduction}

Extracting geometric features like perimeter, area or curvature has an important role in the field of pattern recognition. The applications are various from pattern matching to pattern analysis like for example, discrimination of similar handwriting numerals [5].

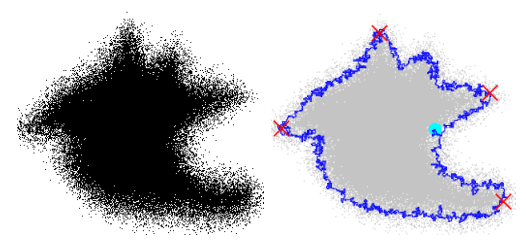

Recently, three new methods were proposed to compute curvature on noisy discrete data. First Nguyen et al. extend the estimator of osculating circles proposed by Coeurjolly et al. [6] by using blurred segments [1]. By this way, the estimation of osculating circles had a better behavior on noisy contours and was also meaningful for a non connected (but ordered) set of points. Then a second approach, proposed by Kerautret and Lachaud [2], suggests to minimize curvature while satisfying geometric constraints derived from tangent directions computed on the discrete contour. The robustness to noise of this approach was given also by blurred segments but used in a different manner. The third curvature estimator, proposed at the same time by Malgouyres et al. [3], suggests to use discrete binomial convolution to obtain a convergent estimator adapted to noisy data.

In this work, we propose to evaluate experimentally these three estimators by using different test contours and by measuring the precision of the estimation

* This work was partially funded by the ANR project GeoDIB, ${ }^{\circ}$ ANR-06-BLAN0225. Bertrand Kerautret was partially funded by a BQR project of Nancy University. 
with several grid sizes. After comparing the curvature obtained with low and large resolution, we measure the robustness of the estimator on noisy contours. A second objective of this paper is to apply the most stable and robust of these estimators to a concrete application of corner detection (see the previous figure). Moreover we compare the obtained results with a recent morphological corner detector [4].

This paper is organized as follows. In the following section, after introducing briefly some notions of digital blurred segments, we give a rapid description of the three estimators. Then the comparison of these estimators is given in section 3. Section 4 shows the application to corner detection and describes the comparative evaluation.

\section{Discrete Curvature Estimators}

Before describing the curvature estimators previously mentioned, we address the notion of digital blurred segments used by the two first estimators.

Blurred segments were introduced by Debled et al. [7]. Note that a comparable algorithm was proposed by Buzer [8]. The notion of blurred segments is based on the arithmetic definition of digital straight line and uses the computation of the convex hull to determine its vertical geometric width $\nu$. From this definition the authors proposed an algorithm for the recognition of blurred segments of width $\nu$. Note that the two following curvature estimators use the version of the algorithm which is not restricted to the hypothesis that points are added with increasing $x$ coordinate (or $y$ coordinate). The next floating figure illustrates two blurred segments of width 5 computed from the central black pixel.

\subsection{Estimator based on osculating circles (CC and NDC)}

The curvature estimator proposed by Nguyen and Debled-Rennesson [7] follows the same concept than the estimator proposed by Coeurjolly et al. [6] (called CC estimator). The latter is based on the estimation of osculating circles. More precisely, by denoting $C$ a discrete curve, $C_{i, j}$ the sequence of

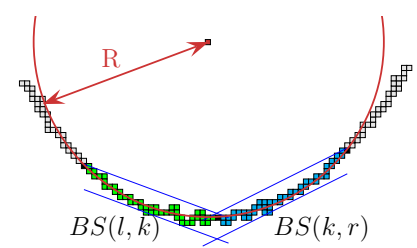
points going from $C_{i}$ to $C_{j}$ and $B S_{\nu}(i, j)$ the predicate " $C_{i, j}$ is a blurred segment of width $\nu$ ". We consider the points $C_{r}$ and $C_{l}$ defined such that: $B S_{\nu}(l, k) \wedge$ $\neg B S_{\nu}(l-1, k) \wedge B S_{\nu}(k, r) \wedge \neg B S_{\nu}(k, r+1)$. With this definition, they determine the curvature of width $\nu$ at the point $C_{k}$ from the radius of the circle passing through $C_{k}, C_{l}$ and $C_{r}$. By noting $s_{1}=\left\|\overrightarrow{C_{k} C_{r}}\right\|, s_{2}=\left\|\overrightarrow{C_{k} C_{l}}\right\|$ and $s_{3}=\left\|\overrightarrow{C_{l} C_{r}}\right\|$, the authors give the following expression of radius $R_{\nu}$ of the circle associated to the point $C_{k}$ :

$$
R_{\nu}\left(C_{k}\right)=\frac{s_{1} s_{2} s_{3}}{\sqrt{\left(s_{1}+s_{2}+s_{3}\right)\left(s_{1}-s_{2}+s_{3}\right)\left(s_{1}+s_{2}-s_{3}\right)\left(s_{2}+s_{3}-s_{1}\right)}}
$$

If the vectors $\overrightarrow{C_{k} C_{l}}$ and $\overrightarrow{C_{k} C_{l}}$ are not collinear then the curvature of width $\nu$ can be determined with $\frac{\operatorname{sign}\left(\operatorname{det}\left(\overrightarrow{C_{k} C_{r}, C_{k} C_{l}}\right)\right)}{R_{\nu}\left(C_{k}\right)}$. Otherwise the curvature is set to 0 . 


\subsection{Global Minimization Curvature estimator (GMC)}

The estimator proposed by Kerautret and Lachaud is based on two main ideas [2]. The first one is to take into account all the possible shapes that have the same digitization defined by the initial contour and to select the most probable contour. This selection is done with a global optimization by minimizing the squared curvature. From this idea, we can expect a precise result even with a low contour resolution. The second idea is to obtain an estimator not sensitive to noise or to non perfect digitization process with blurred segments.

For the purpose of minimizing curvature, the tangential cover [9] is computed on the discrete contour and the minimal and maximal possible tangent values are defined for each pixel. Fig. 1 illustrates both tangential cover (a) and bounds on the tangent directions (b). Minimizing the curvature consists in moving each point $\left(x_{i}, y_{i}\right)$ along the $y$ axis and between the bounds defined by $y_{\min }, y_{\max }$ in order to minimize the slope of the line joining $\left(x_{i}, y_{i}\right)$ to $\left(x_{i+1}, y_{i+1}\right)$. The global minimization is applied with a relaxation process.

For the robustness to noise and non perfect digitization process, the discrete maximal segments were replaced by the discrete maximal blurred segments previously described. Note that the definition of the minimal and maximal slopes of the blurred segments is notably different from the non blurred case (see [2] for more details).

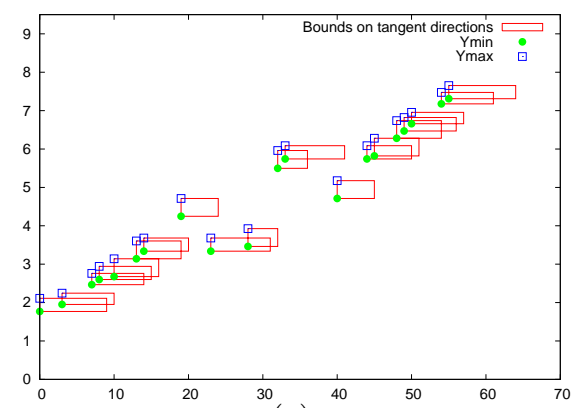

(a)

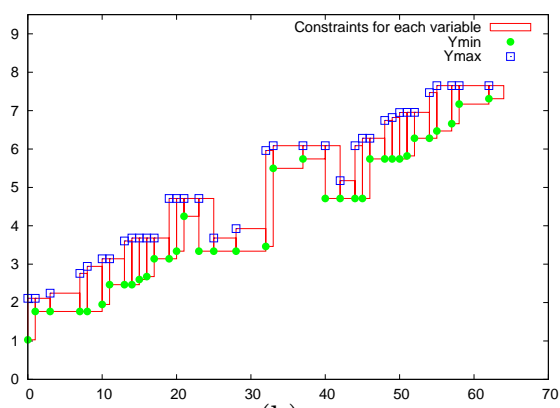

(b)

Fig. 1. Illustration of bounds defined from maximal segments (a) (extracted from the previous floating figure). (b) shows the constraints defined on each variable.

\subsection{Binomial Convolution Curvature Estimator (BCC)}

Malgouyres et al. proposed an algorithm to estimate derivatives with binomial convolutions [3] which is claimed to be multigrid convergent. They define the operator $\Psi_{K}$ which modifies the function $F: \mathbb{Z} \rightarrow \mathbb{Z}$ by the convolution with 
kernel $K: \mathbb{Z} \rightarrow \mathbb{Z}$. For instance the backward finite difference is given by $\Psi_{\delta} F$ where the kernel $\delta$ is defined by:

$$
\delta(a)=\left\{\begin{array}{c}
1 \text { if } a=0 \\
-1 \text { if } a=1 \\
0 \text { otherwise }
\end{array}\right.
$$

Then the authors give the smoothing kernel defined as:

$$
H_{n}(a)=\left\{\begin{array}{cl}
\left(\begin{array}{c}
n \\
a+\frac{n}{2}
\end{array}\right) & \text { if } n \text { is even and } a \in\left\{-\frac{n}{2}, \ldots, \frac{n}{2}\right\} \\
\left(\begin{array}{c}
n \\
a+\frac{n+1}{2}
\end{array}\right) & \text { if } n \text { is odd and } a \in\left\{-\frac{n+1}{2}, \ldots, \frac{n-1}{2}\right\} \\
0 & \text { otherwise. }
\end{array}\right.
$$

Finally the derivative kernel $D_{n}$ is given by $D_{n}=\delta * H_{n}$ and the derivative estimator is $\frac{1}{2^{n}} \Psi_{D_{n}} F$. An interesting point of the method is that higher order derivatives have similar expressions:

$$
D_{n}^{2}=\delta * \delta * D_{n}
$$

And for the curvature we compute:

$$
\frac{\left.D_{n}^{2}(x) * D_{n}(y)-D_{n}^{2}(y) * D_{n}(x)\right)}{D_{n}(x)^{2}+D_{n}(y)^{2}}
$$

The value of $n$ is defined by $n=\left\lfloor h^{2(\alpha-3) / 3}\right\rfloor$, where $h$ represents the grid size and $\alpha \in] 0,1]$ is an additional parameter which can be associated to the amount of noise. More precisely, a value close to 0 allows more noise than a value close to 1.

\section{Experimental Comparisons}

The objective is to measure the precision obtained with the previously described estimators and to give execution times. For this purpose several data sets were generated with different grid sizes $(h)$, for coarse $(h=1)$, medium $(h=0.1)$ and fine resolution $(h=0.01)$. Fig. 2 illustrates the two shapes defined with a grid step equals to 1 (images (a) and (b)). The choice of these test shapes can be justified since it allows to analyse the performances of the estimators both on smooth shapes with quick turns and on polygonal shapes with corners.

We have applied the three estimators on the previous data. For the BCC estimator, the parameter $\alpha$ was set to 1 and the value of $n$ was thus set to $h^{-4 / 3}$. For the other estimators, no parameters were used. From the resulting curvature graphs of Fig. 2, it can be seen that for both the flower and the polygon, the BCC estimator shows numerous oscillations with coarse grid sizes $(\mathrm{h}=1$ and $h=0.1$ ). But the finer the grid size is, the more the values of curvature of the BCC estimator are stable. It appears that it is not the case with the CC estimators. Indeed the oscillations appear to be more important with fine grid size. For the 
GMC estimator we can see that it is very stable since no oscillations are visible. From the squared curvature errors, there are no superior estimators for all test shapes and each estimator has a preferred type shape.

Execution times were measured on these curvature estimations (Tab. 1). On average we can see that the CC estimator is faster than GMC or CC estimator. An exception can be seen for the results obtained on the polygon, since GMC is faster than CC and BCC. It is simply due to the fact that the optimisation process is performed only on points on the frontier of constant tangential cover areas (see Fig. 1). And for a polygon this number of variable is largely smaller than on a circular shape.

\begin{tabular}{|c|c|c|c|c|c|c|c|c|c|}
\hline shape & \multicolumn{3}{|c|}{ Flower } & \multicolumn{3}{c|}{ Circle } & \multicolumn{3}{c|}{ Polygon } \\
\hline$h$ & 1 & 0.1 & 0.01 & 1 & 0.1 & 0.01 & 1 & 0.1 & 0.01 \\
\hline \hline CC & 0.0945 & 0.0225 & 0.0079 & 0.0005 & 0.0009 & 0.0013 & 0.0004 & 0.0003 & $8.1 \mathrm{e}-05$ \\
\hline GMC & 0.0966 & 0.0346 & 0.0049 & $2.5 \mathrm{e}-07$ & $3.2 \mathrm{e}-10$ & $4.3 \mathrm{e}-08$ & 0.0113 & 0.3089 & 3.428 \\
\hline $\mathrm{BCC}$ & 0.0855 & 0.0185 & 0.0081 & 0.0178 & 0.0012 & 0.0001 & 0.0232 & 0.0261 & 0.0510 \\
\hline \hline $\mathrm{CC}_{(\mathrm{ms})}$ & 6 & 84 & 891 & 6 & 55 & 637 & 8 & 82 & 870 \\
\hline $\mathrm{GMC}_{(\mathrm{ms})}$ & 0 & 75 & 2593 & 2 & 363 & 2673 & 0 & 4 & 67 \\
\hline $\mathrm{BCC}_{(\mathrm{ms})}$ & 0 & 18 & 4514 & 0 & 14 & 3275 & 0 & 18 & 4501 \\
\hline
\end{tabular}

Table 1. Mean squared curvature error (upper part of tabular) and execution times obtained with the estimators applied on shapes of Fig. 2 and on a circle of radius 20 (lower part of tabular).

In order to measure the stability of the curvature estimators on noisy shapes, noise was added according to the model proposed by Kanungo [10]. Fig. 3 shows such noise addition (images (a) and (b)). From these results, we can see that the GMC estimator is very stable compared to the NDC estimator. Even with width 2 the results are stable if we expect a small part with negative curvature (image (f)). In the same way, the results of the BCC estimators appear satisfying even if a large size $n$ was needed to obtain stable values. The fact that there are no oscillation constitutes a real advantage to design a simple and efficient curvature based-algorithm. In the following, we therefore exploit the stability of the GMC estimator in order to implement a robust corner detector.

\section{Application to Corner Detection}

We introduce a simple algorithm to detect corner points. The main steps of the algorithm for convex corner points are:

1. for all contour points $\left(p_{i}\right)_{i \in I}$ compute curvature $\kappa\left(p_{i}\right)$ with the GMC estimator with width $\nu$.

2. detect all the maximal curvature regions defined by sets of consecutive points:

$$
\begin{aligned}
R_{k}= & \left\{\left(p_{i}\right)_{i \in[a, b]} \mid \forall i, \kappa\left(p_{i}\right)=\kappa\left(p_{a}\right) \wedge \kappa\left(p_{a-1}\right)<\kappa\left(p_{a}\right) \wedge \kappa\left(p_{b+1}\right)<\kappa\left(p_{b}\right)\right. \\
& \left.\wedge \kappa\left(p_{a}\right)>0\right\}
\end{aligned}
$$




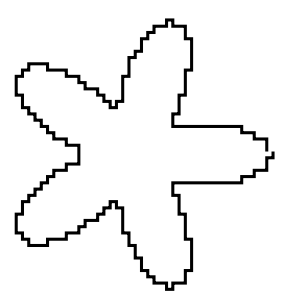

(a)

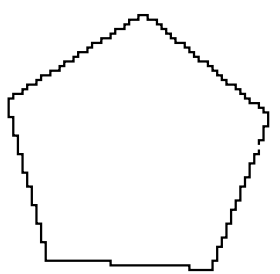

(b)

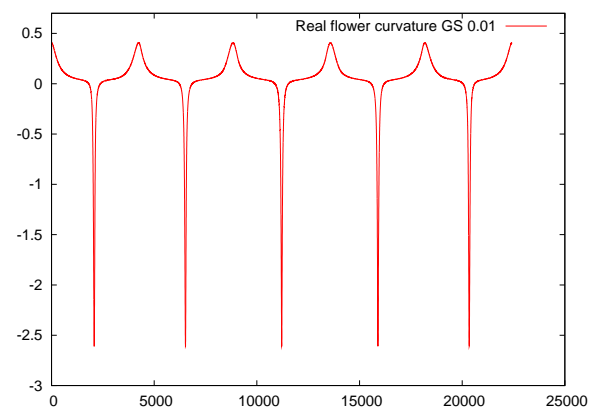

(c)

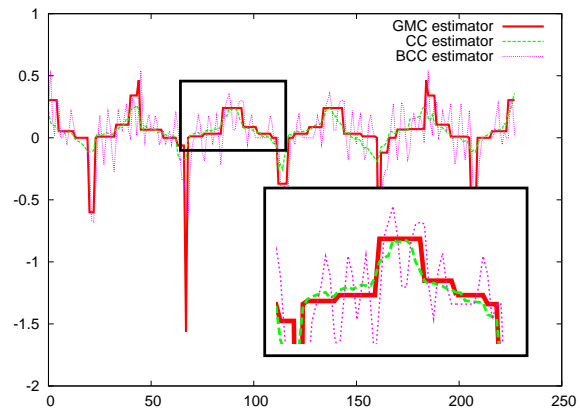

(d) grid size $=1$

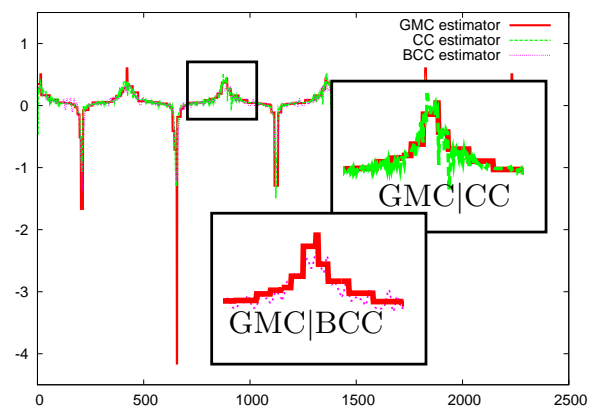

(f) grid size $=0.1$

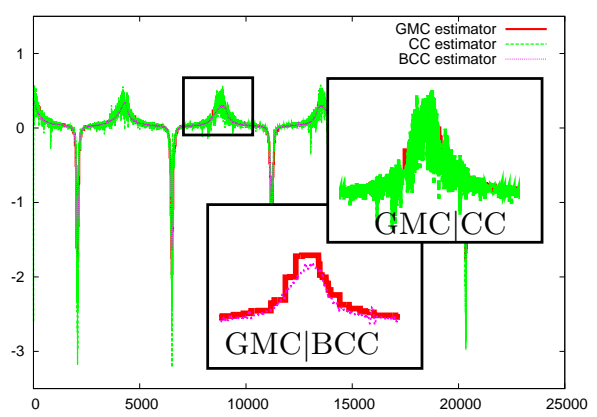

(h) grid size $=0.01$

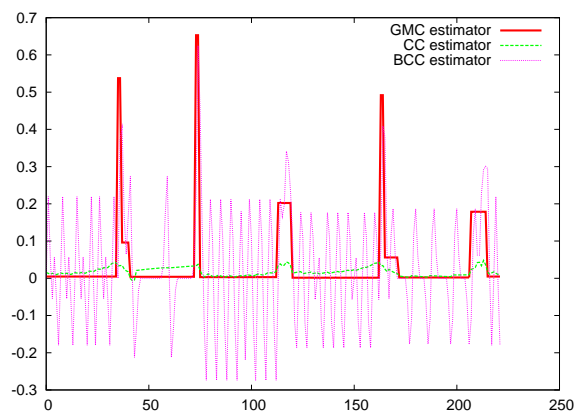

(e) grid size $=1$

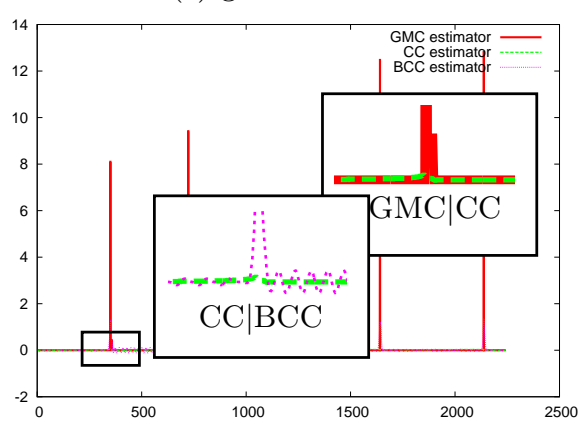

(g) grid size $=0.1$

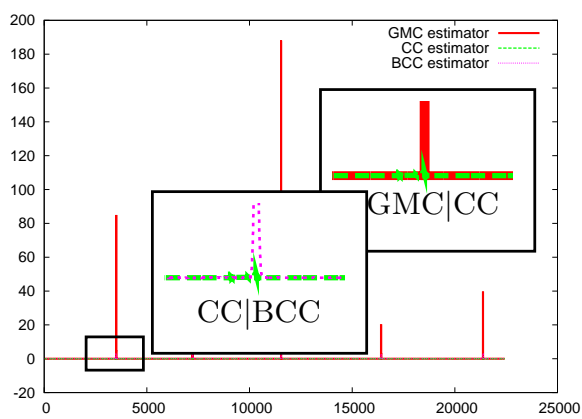

(i) grid size $=0.01$

Fig. 2. Comparisons of the CC, GMC and BCC estimators on the flower (a,d,f,h) and on the polygon (b,e,g,i). (c) shows the real curvature associated to the flower shape. 


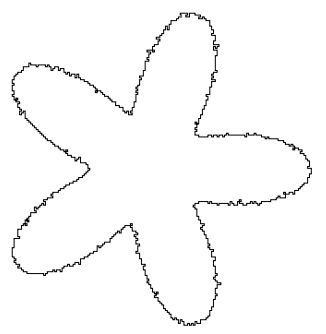

(a)

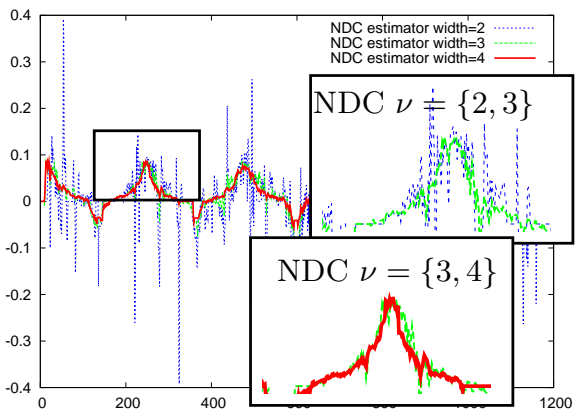

(c) NDC estimator

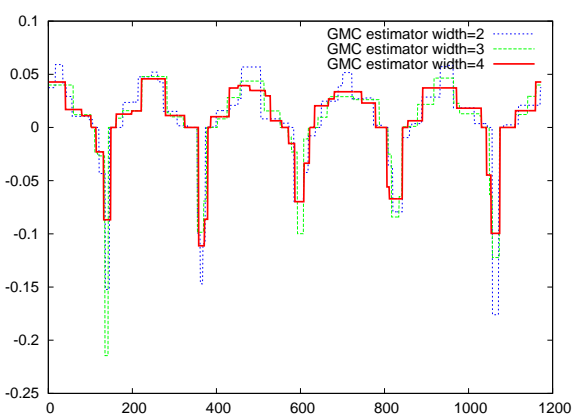

(e) GMC estimator

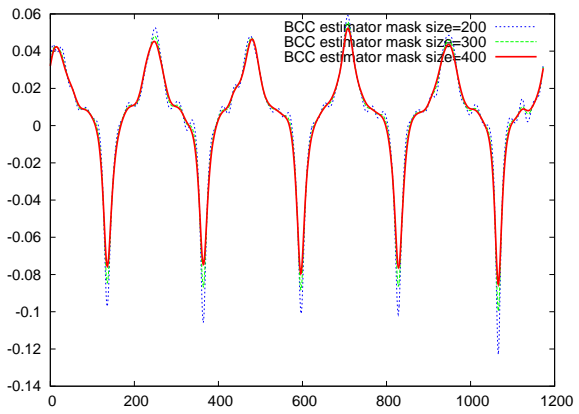

(g) BCC estimator

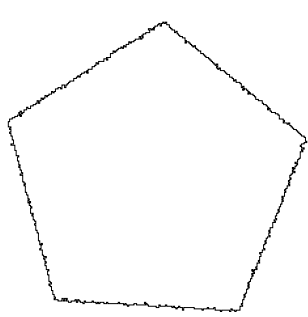

(b)

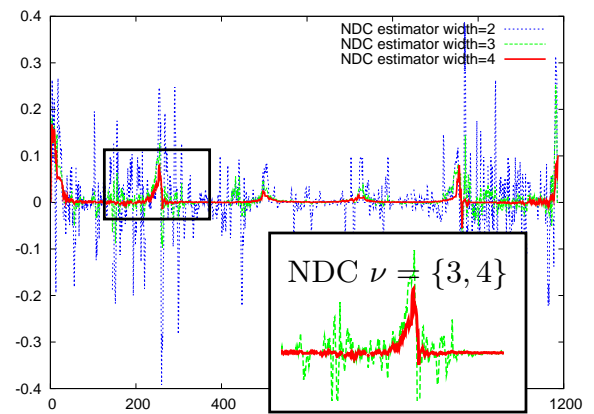

(d) NDC estimator

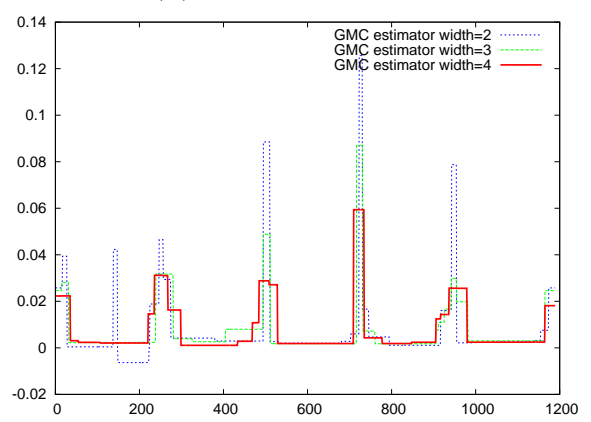

(f) GMC estimator

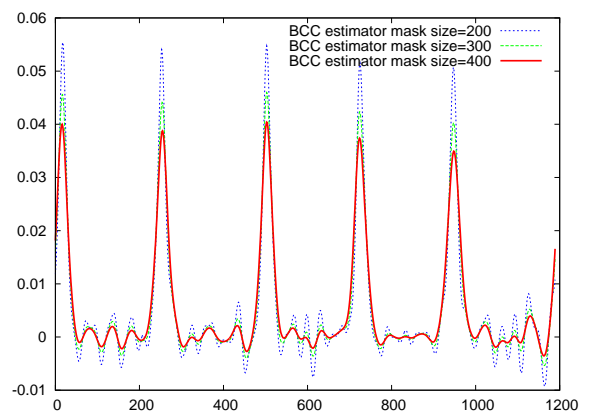

(h) BCC estimator

Fig. 3. Results obtained on the noisy version on the shapes of figure 2. Each row shows the result obtained for each estimator used with different parameters associated to the amount of noise (width $\nu$ for GMC et NDC and mask_size for the BCC estimator) . 
3. for each region $R_{k}$ mark the point $p_{(a+b) / 2}$ as a corner.

4. (optional) select only corner points with curvature bigger than a threshold value $\kappa_{\min }$.

Note that a quantification of the curvature field was applied in order to simplify the detection of the local minima/maxima (set to $1 e-3$ ). In the rest of this paper we have not applied the optional step (4) since it adds a new parameter and it could be fixed for each particular application. The concave corner detection is deduced by replacing the maximal by the minimal curvature regions.

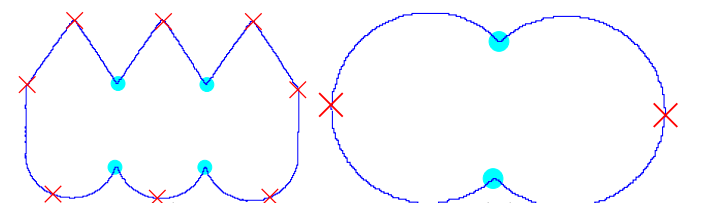

(a)

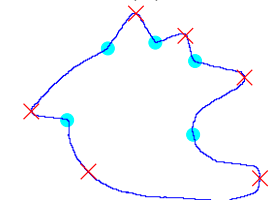

(e) (b)

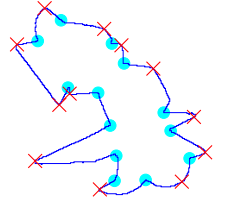

(f)

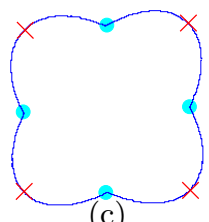

(c)

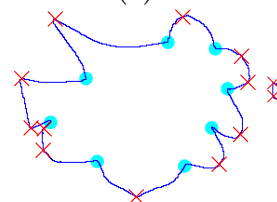

(g)

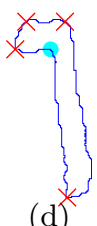

(d)

Fig. 4. Results of corner detection obtained with the GMC estimator (with width $\nu=2$ ). Convex and concave corners are respectively represented by crosses and disks.

The previous algorithm was applied on the standard image collection available on-line [11] (see Fig. 4). All the corner detections were performed with the GMC estimators with the standard width $\nu=2$ which is an usual value for real data presenting a weak amount of noise. We can see on Fig. 4 that some parts are considered as corner which are only local minima. Note that points could be easily removed by applying a small threshold on the curvature values of the local maxima. For this standard dataset our results are comparable to previous works, but we can note that we have only one parameter which can be unchanged for all data considered as non noisy. This is not the case for example for corner detector proposed by [4] as described below.

The images of Fig. 5 were generated from the previous test images and with the same noise model as mentioned in the previous section. The value of the width $\nu$ used for the curvature estimation was set to 4 according to the quantity of noise. Note that we are actually working on an automated determination of this parameter. Despite the important amount of noise, the corners were well detected. The only defect can be seen on the left wing of the plane (h), but it disappears when using a width equals to 5 . Even with a lot of noise, corners can be detected as illustrated on the introducing figure of page $1(\nu=13)$. Note that the total execution time was approx. 3s for the test images of Fig. 4 and Fig. 5.

We have compared our new corner detector with a recent morphological method identified as BAP (Base Angle Points) [4] which shows good performance in comparison with other techniques of the literature. This method is based on 


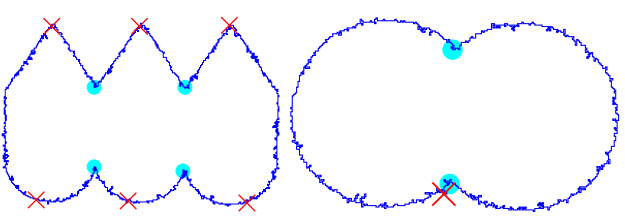

(a)

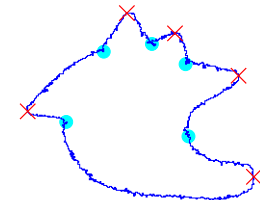

(e) (b)

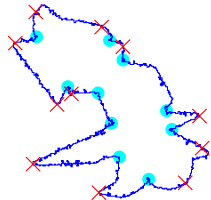

(f)

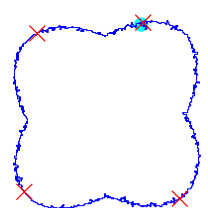

(c)

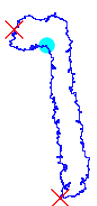

(d)

Fig. 5. Results obtained on noisy version of the original test images. The width $\nu$ of the GMC estimator was set to 4 for all the images.

the analysis of the residuals of an opening of the original image considered as a set $I$ with a disk structuring element of size $\lambda^{3}$ :

$$
C(I)=I \backslash \gamma_{B_{\lambda}}(I),
$$

where $\gamma_{B}(X)=\cup_{i}\left\{B_{i} \mid B_{i} \subseteq X\right\}$. These components contain potential corner points. Some filtering can be performed to suppress irrelevant components (using a size criterion for example). For each residual component is computed an isosceles triangle composed of two base points $B, C$ and of the corner point $A$. The length $L$ of the base segment $|B C|$ and the height $H$ of the triangle can be used to construct a filter which discriminates the true corners from noisy parts.

We have experimented this technique using three parameters: the radius $\lambda$ of the disk structuring element, and the minimal values $L$ and $H$ for each residual component. Fig. 6 show the results obtained with the BAP method on the noisy shapes. For each experiment, despite the manual tune of these parameters to obtain the best possible results, the results are not convincing on noisy data compared to our proposed method.

\section{Conclusion}

We have compared several recent discrete curvature estimators adapted to noisy discrete data. The mean error obtained on three sorts of shape does not discriminate a special estimator since for each shape a specific estimator gives the smallest mean error. For execution time, the CC estimator is faster than the other. On the point of view of stability the GMC estimator shows few oscillation even on noisy data. After the evaluation of this estimators we have proposed to apply the most stable estimator to corner detection. The obtained results show very important robustness to noise and outperform other recent corner detectors. In future works we will perform a global comparison of our corner detector with other existing works.

\footnotetext{
${ }^{3}$ This operator is called white top-hat [12].
} 


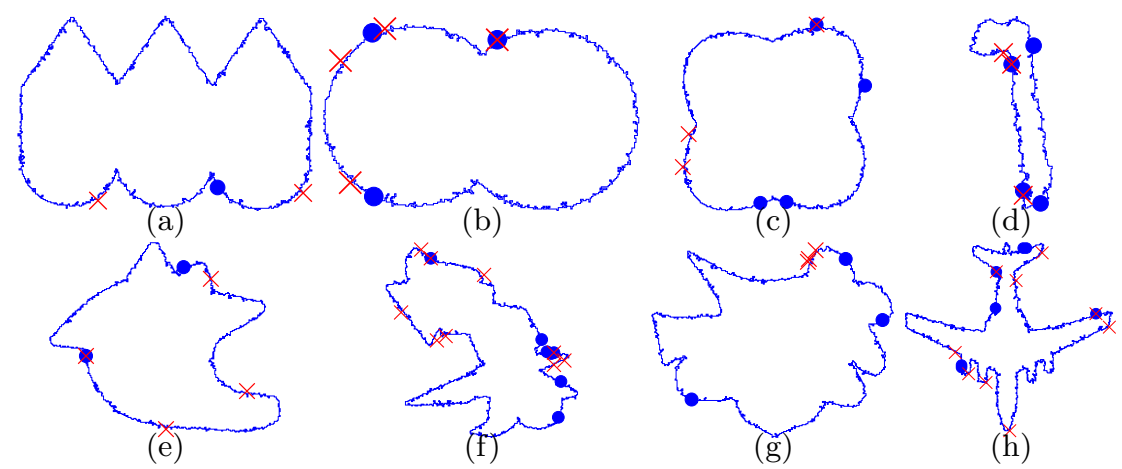

Fig. 6. Results obtained with BAP algorithm on the noisy version of the original test images. The crosses and circles represent respectively corner points detected with parameters $(\lambda, L, H)=(2,2,1)$ and $(\lambda, L, H)=(3,1,1)$.

\section{References}

1. Nguyen, T., Debled-Rennesson, I.: Curvature estimation in noisy curves. In: CAIP. Volume 4673 of LNCS., Springer (2007) 474-481

2. Kerautret, B., Lachaud, J.: Robust estimation of curvature along digital contours with global optimization. In: Proc. of Int Conf DGCI. Volume 4992 of LNCS., Springer (2008) 334-345

3. Malgouyres, R., Brunet, F., Fourey, S.: Binomial convolutions and derivatives estimations from noisy discretizations. In: Proceedings of the Int Conf on DGCI. Volume 4992 of LNCS., Springer (2008) 370-379

4. Chang, X., Gao, L., Li, Y.: Corner detection based on morphological disk element. In: Proceedings of the 2007 American Control Conference, IEEE (2007) 1994-1999

5. Yang, L., Suen, C.Y., Bui, T.D., Zhang, P.: Discrimination of similar handwritten numerals based on invariant curvature features. Pat. Rec. 38 (2005) 947-963

6. Coeurjolly, D., Miguet, S., Tougne, L.: Discrete curvature based on osculating circle estimation. In: Proc. Int. workshop Visual Form. Volume 2059 of LNCS., Springer (2001) 303-302

7. Debled-Rennesson, I., Feschet, F., Rouyer-Degli, J.: Optimal blurred segments decomposition of noisy shapes in linear times. Computers and Graphics (2006)

8. Buzer, L.: An elementary algorithm for digital line recognition in the general case. In: Proc. of Int. Conf. DGCI. Volume 3429 of LNCS., Springer (2005) 299-310

9. Feschet, F., Tougne, L.: Optimal time computation of the tangent of a discrete curve: Application to the curvature. In: Proc. of the Int Conf DGCI. Volume 1568., springer (1999) 31-40

10. Kanungo, T.: Document Degradation Models and a Methodology for Degradation Model Validation. PhD thesis, University of Washington (1996)

11. Chetverikov, D., Szabo, Z.: http://visual.ipan.sztaki.hu/corner/corner click.html. Online (1999)

12. Soille, P.: Morphological Image Analysis: Principles and Applications. SpringerVerlag Berlin Heidelberg (2003) 